\title{
FAMILIAL (IDIOPATHIC) SCOLIOSIS
}

\author{
A Family Survey \\ Ruth Wynne-Davies, Edinburgh, Scotland \\ From the Medical Research Council Research Group on Genetic Problems in Orthopaedic Disease, \\ Department of Orthopaedic Surgery, Edinburgh University
}

The diagnosis of idiopathic scoliosis is made after other known causes of the deformity have been excluded. This paper does not deal with scoliosis resulting from vertebral anomalies, nor with that associated with conditions of known genetic etiology, such as neurofibromatosis, Marfan's syndrome or muscular dystrophy. Also, the diagnosis of idiopathic scoliosis is only made when the curve is structural, that is, when vertebral rotation accompanies

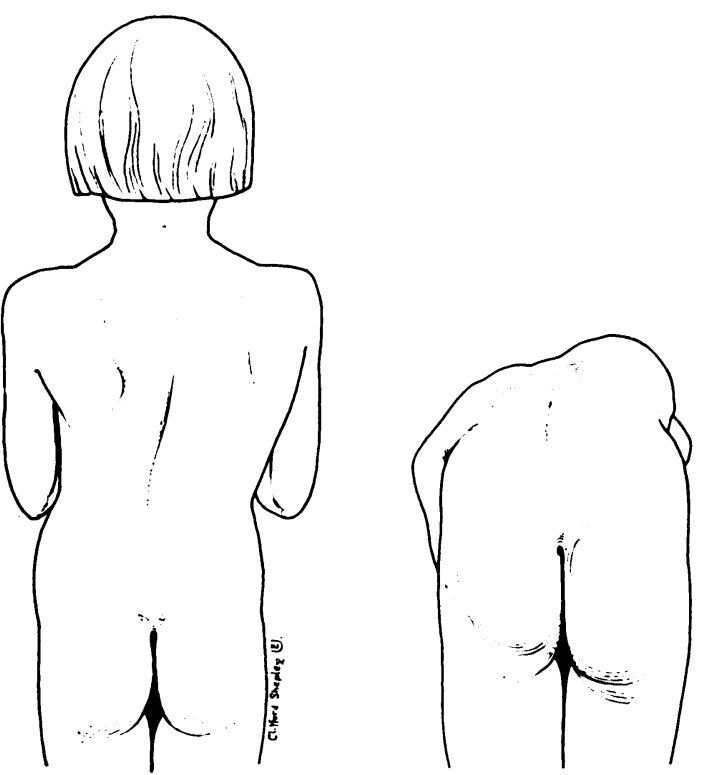

Fig. 1

Right thoracic curve with rib hump, indicating the vertebra rotation of structural scoliosis. the lateral curve. This is a clinical feature, made evident as the individual bends forwards to touch his toes, when a " rib hump" appears (Fig. 1).

The aim of this study was to establish the family incidence of the deformity in 114 patients and to compare this with the incidence in the general population. This would indicate if there is a concentration of the deformity within families and would give some guide as to the nature of any genetic factor involved. In addition, the patients were subdivided by age of onset of the curve, whether the deformity resolved or progressed, and whether it was accompanied by any other developmental anomaly. By comparing the figures from the family survey in each of these groups it is possible to obtain some evidence as to whether these differing clinical patterns share the same basic etiology.

\section{MATERIAL AND METHOD}

A total of 180 case records from the Edinburgh Scoliosis Clinic were analysed. One hundred and sixty-one individuals with idiopathic scoliosis as the only deformity were noted, and nineteen with scoliosis together with some other (non-vertebral) defect. Eight further patients with scoliosis and some associated defect were added from the orthopaedic department of the Royal Hospital for Sick Children, Glasgow, by courtesy of Mr N. J. Blockey. One hundred and fourteen of these index cases were selected and the families of each of them (the first, second and third degree relatives) were personally examined for a " rib hump." This is not sufficient for a firm diagnosis of idiopathic scoliosis because it is present in all patients with structural scoliosis whatever the etiology; but it is a useful method of rapid screening. Individuals with this physical sign were then brought to their nearest hospital for a more detailed examination and radiography. This work entailed travelling all over the British Isles, seeing well over 2,000 individuals. The project was started in 1962 by Mr R. S. M. Ling who collected the first thirty-eight pedigrees. 
Age distribution and side of curve-These were similar to those described by James, LloydRoberts and Pilcher (1959). The typical curve in the infant patient was left thoracic ( 88 per cent), and in the adolescent right thoracic ( 90 per cent), with a change-over in the middle years of childhood.

There are two peaks of incidence, one in infancy and the other in adolescence (Fig. 2). The deformity is rarely apparent at birth, but develops during the first year of life. There are very few new cases in the middle, juvenile years, but there is a big increase during adolescence, almost exclusively in girls. Two main groups were therefore available for comparison: early onset scoliosis (under eight years) and late onset (eight years and over). The early onset type might be "resolving" or "progressive." Once growth ceases the deformity becomes static.

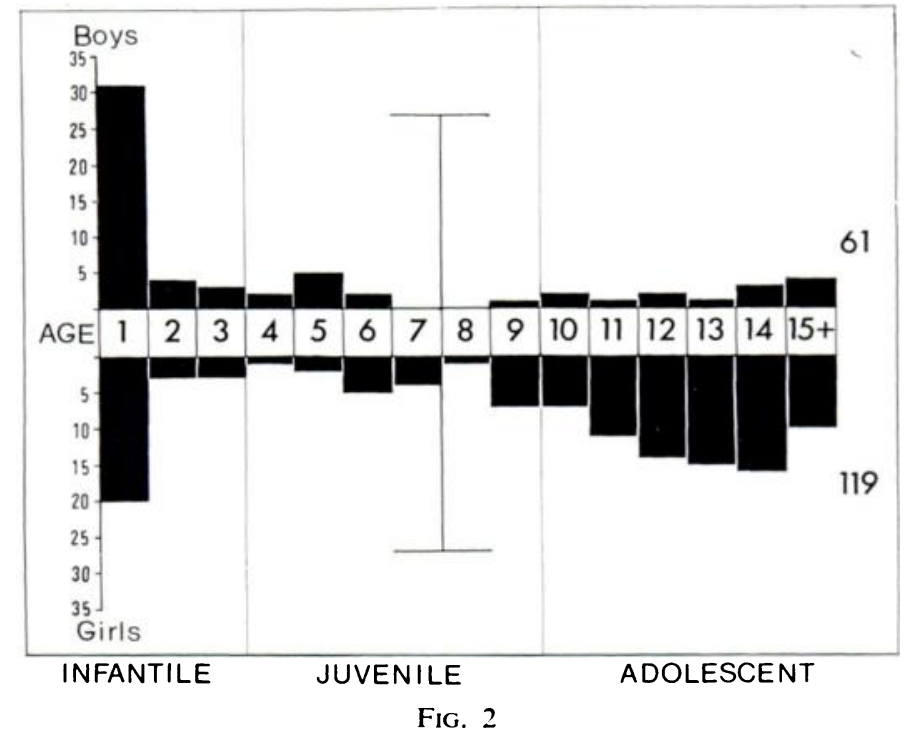

Age of onset of idiopathic scoliosis in 180 patients.

It is of interest that the infantile form of scoliosis is very uncommon in North America, where attendances at scoliosis clinics consist almost entirely of adolescent cases. Figures are available from lowa (Ponseti 1966) where the number presenting under eight years of age is only 8 per cent of the total compared with 50 per cent in Britain.

Sex ratio-In the early onset group there is a slight excess of boys over girls $(1 \cdot 21$, or approximately five boys to four girls). Late onset scoliosis is rare in boys, the ratio being $0 \cdot 15$ (one boy to seven girls).

Correction for age-Because scoliosis could develop at any age up to fifteen years, it was apparent that younger relatives could not, as it were, count their full weight. A graph has, therefore, been constructed from the total number of cases attending the scoliosis clinic, showing the percentage of boys and girls affected at each age (Fig. 3). For example, a young sister aged three years counts as only 0.26 of a person because by this age only 26 per cent of the girls who will develop scoliosis have done so.

Population incidence-It was necessary to do a population survey, because from the beginning of the family survey it was apparent that many individuals had a mild degree of scoliosis without being aware of it, and the figures were thus unreliable until the general population incidence was established. This survey was done in Edinburgh schools, nursery schools and infant clinics and included children from two weeks to eighteen years of age. The same criteria were used for diagnosis, and children with a rib hump were brought to hospital for

VOL. 50 B, NO. 1, FEBRUARY 1968 
further examination and radiography. The results (Table I) were in reasonable agreement with the proportions observed in the scoliosis clinic; only one child was found with resolving scoliosis.

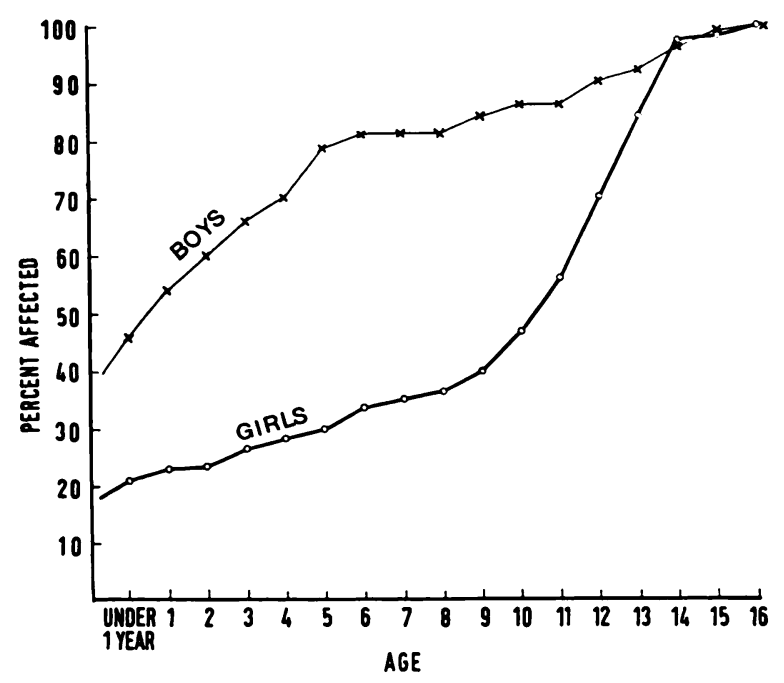

Fig. 3

Percentage of boys and girls affected at each age by idiopathic scoliosis.

\section{RESULTS}

Family survey-There were ninety-two index patients with scoliosis as the only deformity (including thirteen with resolving scoliosis), and twenty-two with some other anomaly in addition (including one resolving case). Sixty-one were of the early onset type and fifty-three late onset.

Of the 114 index patients investigated, thirty-one (about one-quarter) had one or more affected individuals in the family. The incidence of scoliosis amongst relatives in all groups of index patients was considerably in excess of the population rate.

TABLE I

Idiopathic Scoliosis SuRvey: 11,087 Edingurgh ChILDREN

\begin{tabular}{|c|c|c|c|}
\hline $\begin{array}{c}\text { Number of } \\
\text { children }\end{array}$ & \multicolumn{2}{|c|}{ Population incidence per 1,000 } \\
\cline { 2 - 3 } & Male & Female & Total \\
\hline $\begin{array}{c}\text { Early onset } \\
(3,193)\end{array}$ & $\begin{array}{c}1 \cdot 2 \\
(2 \text { of } 1,653)\end{array}$ & $\begin{array}{c}1 \cdot 3 \\
(1 \text { and } 1 \text { resolving } \\
\text { of } 1,540)\end{array}$ & $1 \cdot 3$ \\
\hline $\begin{array}{c}\text { Late onset } \\
(7,894)\end{array}$ & $\begin{array}{c}0 \cdot 3 \\
(1 \text { of } 3,789)\end{array}$ & $\begin{array}{c}3 \cdot 9 \\
(16 \text { of } 4,105)\end{array}$ & $1 \cdot 8$ \\
\hline
\end{tabular}

Scoliosis and osteoporosis - When the figures of the idiopathic survey were first analysed, it was found that there were more individuals with scoliosis amongst second degree relatives than amongst first degree, which genetically was not explicable. This group was reviewed again and it was then appreciated that many of them were elderly individuals with osteoporosis and a curve. This has formed a subsidiary research project which will be reported in due course.

The histograph (Fig. 4) illustrates the figures for first, second and third degree relatives added, and so overall percentages are low. The highest incidence is among the first degree relatives (parents, siblings and children) of the adolescent girls' group (Table II), and this number is approximately halved in the second and again in those of the third degree; but 
even in these distant relatives the incidence is still four times that of the general population figure of 0.39 per cent.

The highest incidence of all was among the first degree female relatives of these adolescent girl patients, with 12 per cent affected.

The early onset group did not show such a high percentage of affected relatives, and the incidence from first to third degree dropped very little ( $2 \cdot 6$ per cent, $2 \cdot 3$ per cent, 1.4 per cent). However, compared with a population incidence of 0.1 per cent the family figures were clearly increased.

The male and female relatives of male and female index cases were analysed separately, because the sex incidence is unequal in both early and late onset groups. The results indicated only that the groups in which scoliosis is commoner (infant boys and adolescent girls) had more affected relatives than those in which it is less common (infant girls and adolescent boys).

There was an approximately equal incidence amongst parents, siblings and children ( 3 per cent, 5 per cent, 5 per cent).

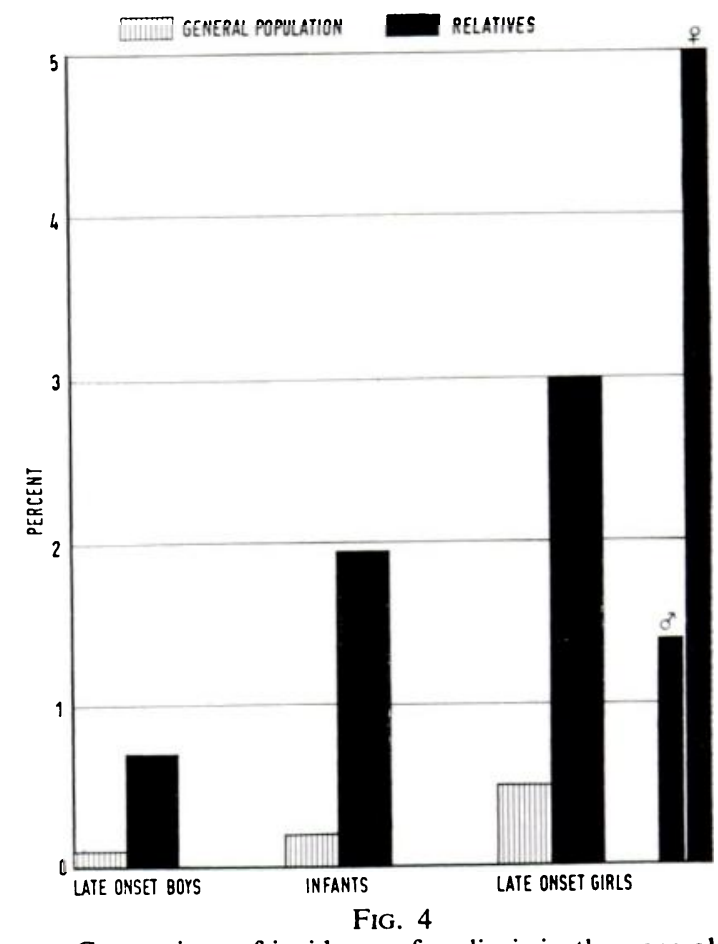

Comparison of incidence of scoliosis in the general population and among the relatives of scoliotic patients.

One of the points that emerged from the survey was that a child who had developed a curve in infancy might have a relation with the adolescent type of curve, and vice versa. However, there were many cases in which there was no information as to age of onset of scoliosis, the individual being unaware of its presence. Indirect evidence was obtained from

TABLE II

Idiopathic Scoliosis-Late OnSET Girls (Forty-two index patients)

\begin{tabular}{|c|c|c|}
\hline \multicolumn{2}{|c|}{ Affected relatives* } \\
\cline { 1 - 2 } First degree & Second degree & Third degree \\
\hline $\begin{array}{c}10 \text { of } 144 \\
\text { (6.94 per cent) }\end{array}$ & $\begin{array}{c}\text { (3.69 of } 217 \\
\text { per cent })\end{array}$ & $\begin{array}{c}\text { (1.55 of } 194 \\
\text { per cent })\end{array}$ \\
\hline
\end{tabular}

* Figures corrected for age.

noting the sex of all the affected relatives, and the side of the curve. In the infantile group of patients the curve is typically in a boy and convex to the left, and in the adolescent group, in a girl and to the right. The pattern among the relatives of each group did not conform with this distribution. Male and female, left and right, approached equal numbers, indicating a probable mixture of infantile and adolescent cases amongst them.

Resolving scoliosis - In this series, of children seen under one year of age, the scoliosis resolved without treatment in half of them, and in half progressed to a more severe deformity. The onset was nearly always during the first six months of life, and the deformity disappeared over the next year or so. In all other respects it was like the progressive type, being nearly always left-sided and rather more common in boys than girls. The evidence for including 
them with the children with a progressive type of curve is that scoliosis is found among their relatives in precisely the same proportions as in the main "early onset " group, and much in excess of the population incidence. This suggests that the resolving type of scoliosis is the mildest manifestation of what may be a very severe disorder.

TABLE III

Maternal Age Distribution in Ninety-four Cases of Adolescent Idiopathic Scoliosis

\begin{tabular}{|c|c|c|c|c|c|}
\hline Age of mother (years) & $15-24$ & 25-29 & $30-34$ & 35 and over & Total \\
\hline Adolescent scoliosis & 22 & 17 & 29 & 26 & 94 \\
\hline \multirow[t]{2}{*}{ Normal population } & $28 \cdot 54$ & $28 \cdot 77$ & $20 \cdot 54$ & $16 \cdot 15$ & $94 \cdot 00$ \\
\hline & \multicolumn{3}{|c|}{$x_{(2)}^{2}=15.81 \quad 0.031<p<0.01$} & & \\
\hline
\end{tabular}

Twins-There was one pair of female twins (probably identical) in the survey, with the adolescent type of scoliosis; one had a left and the other a right lumbar curve. Further investigation was refused, but another sister also had scoliosis. The parents were both dead, but were thought to have been normal.

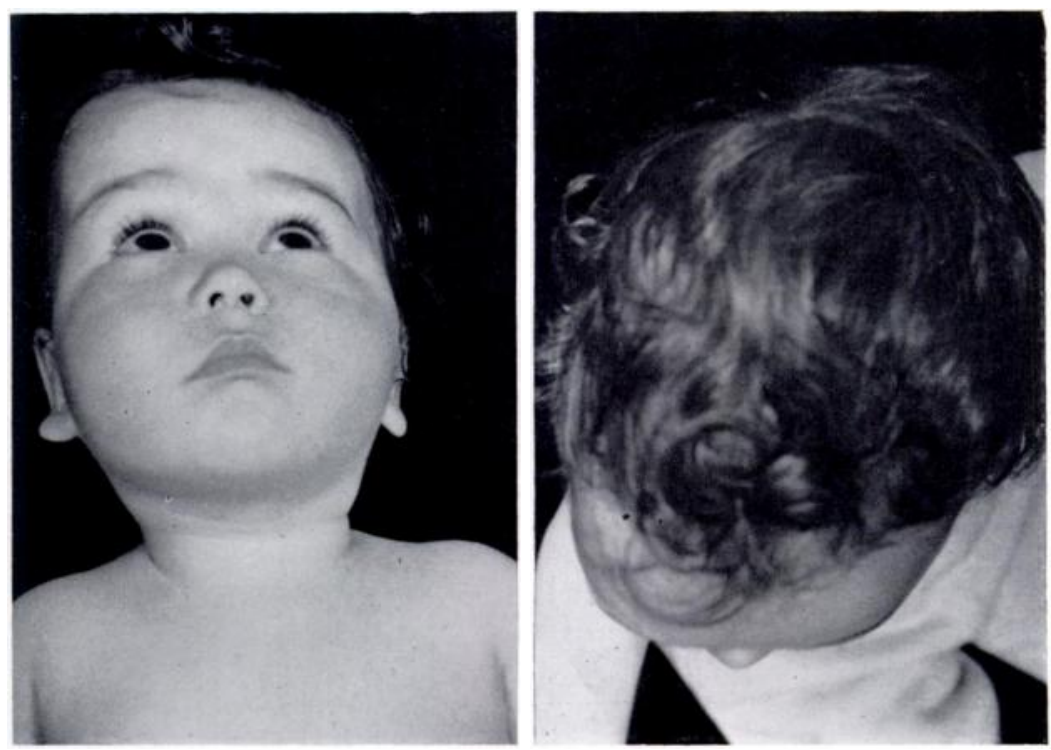

FIG. 5

Left-sided plagiocephaly, accompanied by left thoracic scoliosis.

There were three pairs of dizygotic twins, one only of each pair affected: a girl with adolescent scoliosis with a male twin; a boy with adolescent scoliosis with a male twin: and a boy with infantile resolving scoliosis with a female twin. Another girl with adolescent scoliosis had a male twin who died at birth.

Consanguinity-There was no instance of parental consanguinity.

Parental age and parity-There was a positive finding here in the group of children with adolescent scoliosis. The mother was significantly older than normal by comparison with a control series covering the same years obtained from the Registrar General's figures on parental age. The trend was apparent in the cases in which the detailed family survey was being carried out, and a questionnaire was then sent to a further group in order to increase the numbers (Table III). 
Paternal age and parity showed no significant (independent) difference from normal, and neither was there any maternal age effect in the children with early onset scoliosis.

Associated anomalies. Plagiocephaly-Moulding of the head was present in all infants with scoliosis seen under the age of one year, and was present both in the resolving and progressive types (Fig. 5). The deformity was transient, and by the age of three years only half of them showed it, and by five years there was only the occasional child with a persistent deformity. In all cases the plagiocephaly agreed with the side of the curve (that is $\mathbf{8 8}$ per cent of infants had left-sided curves, and the left side of the head appeared pushed back).

A control group of infants under one year in the city clinics was examined, and seventy-nine of 625 (11 per cent) were found to have plagiocephaly, equally left- and right-sided. Between one and three years 3 per cent were found, but none above this age.

Other associated anomalies - The case records of twenty-seven children with apparent idiopathic scoliosis together with some other developmental defect have been analysed and the families of twenty-two of them visited (Fig. 6).

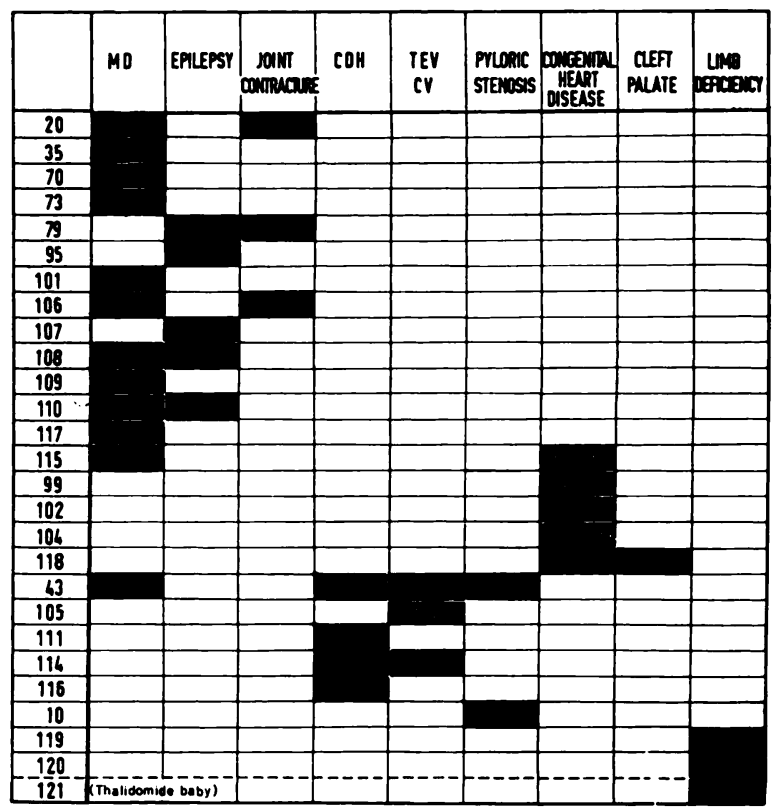

Fig. 6

Anomalies associated with idiopathic scoliosis (twenty-seven patients).

Mental defect and epilepsy, taken together, were the commonest of the associated features (fifteen children). Other cases presented with congenital dislocation of the hip, club foot, various congenital heart disorders, and upper limb defects. One thalidomide child has developed scoliosis.

In a review of the largest group (mental defectives and epileptics), and examination of their families, several relatives were found with uncomplicated idiopathic scoliosis. The proportions of affected relatives were the same as in the main group and much in excess of the population incidence.

The other groups were too small to consider separately, but there was one instance of idiopathic scoliosis in the family of a child with both scoliosis and congenital dislocation of the hip. Associated anomalies in relatives-Apart from the high incidence of scoliosis already noted, developmental anomalies amongst the relatives showed no difference from that which would be expected from a random survey.

VOL. $50 \mathrm{~B}$, NO. 1, FEBRUARY 1968 


\section{DISCUSSION}

There is undoubtedly a concentration of idiopathic scoliosis within the families of index patients. It is probable that the infantile and adolescent types share the same basic etiology, since families contain instances of each, but there are distinct differences between the two groups. In the early onset type the curve is usually to the left, is common in boys, and the deformity resolves in half the cases presenting under one year of age. It occurs only rarely in North America. The number of relatives affected with scoliosis is less than in the following group, and the incidence remains similar in those of the first, second and third degree. The age of the parents is not significantly different from normal.

In the late onset type the curve is usually to the right and in a girl. Although the curve is often severe it may not be so, but it does not disappear spontaneously. There is a very much stronger family history of scoliosis in the girls, but not in the boys with adolescent scoliosis. The incidence in relatives is halved from first to second and then to third degree. The maternal age is significantly increased.

It is probable that there is a strong environmental factor acting in the early onset type. It has been suggested (Browne 1965, Lloyd-Roberts and Pilcher 1965) that intra-uterine compression contributes to the onset of scoliosis in infants, and this at least could account for the constant association of infantile scoliosis and plagiocephaly.

The genetic factor is stronger in the adolescent group and shows some of the characteristics of dominant inheritance. However, the numbers are too small to allow a definite conclusion between this and multiple gene inheritance. The maternal age effect is not understood, but there may be an association between this, the rise in incidence of scoliosis in adolescent girls and the physiological changes at puberty.

\section{SUMMARY}

1. Idiopathic scoliosis is a familial condition.

2. The findings suggest either dominant or multiple gene inheritance, but a larger series is needed before a firm conclusion can be drawn.

3. The infantile and adolescent types of scoliosis seem to share the same basic etiology, because their families contain instances of each.

4. Infants with resolving scoliosis have affected relatives in the same proportions as in the main group, suggesting this is a mild form of the same disorder.

5. In this series all infants seen with scoliosis under one year of age had plagiocephaly, which was usually transient.

6. Mental defect and epilepsy are the commonest findings associated with scoliosis.

7. In adolescent scoliosis the age of the mother is significantly raised by comparison with the expected figure for the normal population.

I am much indebted to Professor J. I. P. James for access to records and patients in his scoliosis clinic and for his great interest and help throughout the study. Acknowledgement and thanks are also due to Dr C. E. Blank and Mrs S. C. Bairstow of the Sheffield Human Genetics Centre for their interest in the maternal age effect in scoliosis and for a detailed analysis of the figures summarised in Table III.

\section{REFERENCES}

Browne, D. (1965): Congenital Postural Scoliosis. British Medical Journal, 2, 565.

James, J. I. P., Lloyd-Roberts, G. C., and Pilcher, M. F. (1959): Infantile Structural Scoliosis. Journal of Bone and Joint Surgery, 41-B, 719.

Lloyd-RoberTs, G. C., and Pilcher, M. F. (1965): Structural Idiopathic Scoliosis in Infancy; a Study of the Natural History of 100 Patients. Journal of Bone and Joint Surgery, 47-B, 520.

PONSETI, I. V. (1966): Personal communication.

Registrar General's RePORT fOR ScOtLand 1939-1963. Her Majesty's Stationery Office. 